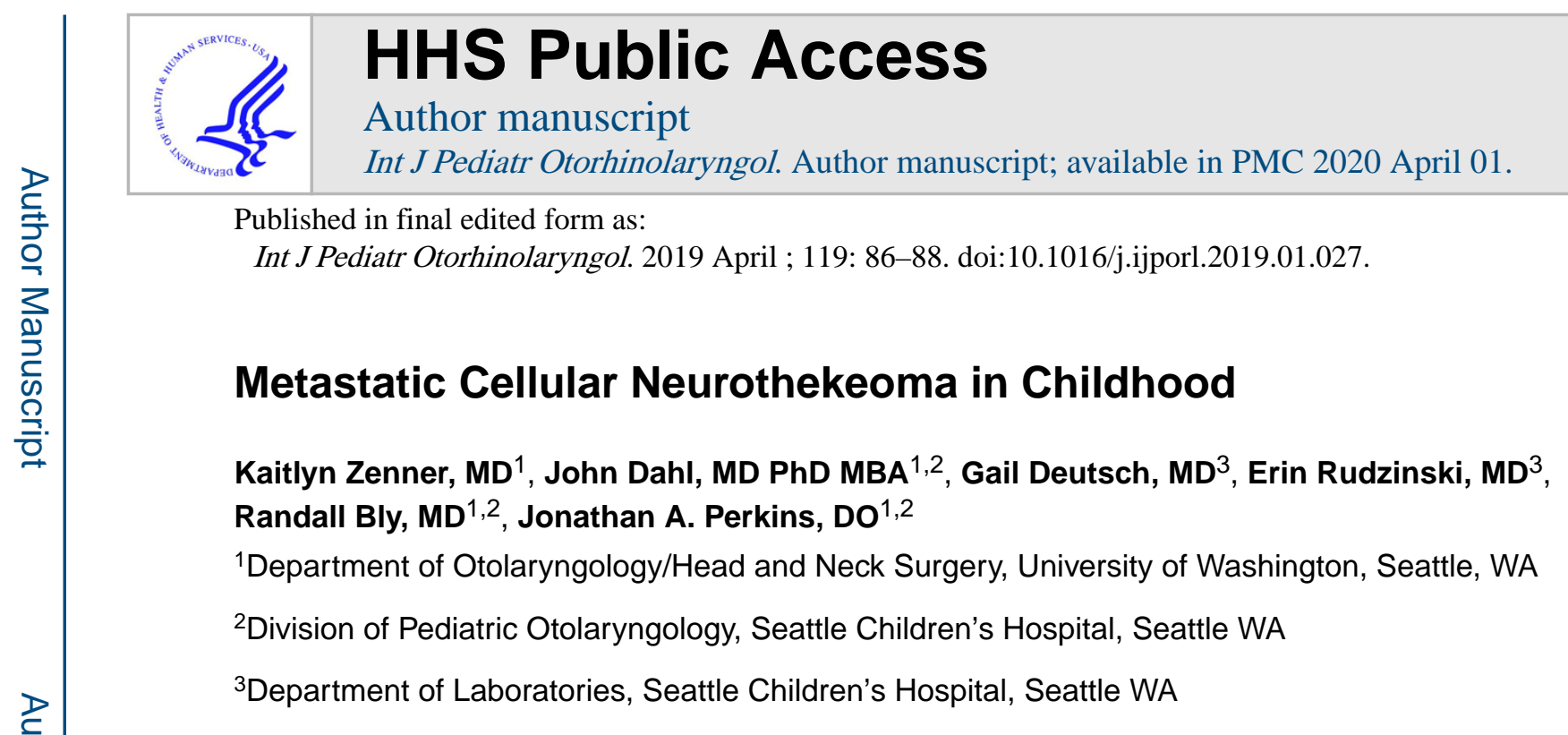

\title{
Introduction
}

Neurothekeoma is a rare cutaneous nerve sheath neoplasm usually presenting in the head and neck region of young females. ${ }^{1,2}$ Although these tumors demonstrate locally aggressive behavior, no cases of metastasis have previously been reported in the literature. Here, we present the case of a 7-year-old male with cellular neurothekeoma of the nasal vestibule with cervical metastasis.

\section{Report of a case}

A 6-year-old healthy male developed an asymptomatic left nasal vestibule mass.

Subsequently, he had occasional self-limited bleeding from the mass. Five months after presentation he underwent resection of the mass. Pathology revealed cellular neurothekeoma with positive margins prompting repeat excision and reconstruction using conchal cartilage. No neck mass was documented at that time and no preoperative imaging was obtained.

One year following initial presentation he developed a firm left submandibular mass and a firm $3 \times 3 \mathrm{~mm}$ nodule was found in the left nasal vestibule at the site of previous resection. Computed tomography showed a $2 \times 3 \mathrm{~cm}$ homogeneous mass in left level 2 neck (Figure 1) and he was referred to Seattle Children's Hospital for further management. The patient, now sixteen months from original presentation, underwent intranasal biopsy, excision of left submandibular mass, and left levels Ib, II, III, and Va neck dissection. During the surgery an erythematous, firm lesion relatively fixed to underlying tissue was noted lateral to the left submandibular gland. The lymph node packet contained multiple enlarged lymph nodes which prompted removal of level Va in addition to levels Ib, II, and III.

\footnotetext{
Publisher's Disclaimer: This is a PDF file of an unedited manuscript that has been accepted for publication. As a service to our customers we are providing this early version of the manuscript. The manuscript will undergo copyediting, typesetting, and review of the resulting proof before it is published in its final citable form. Please note that during the production process errors may be discovered which could affect the content, and all legal disclaimers that apply to the journal pertain.

Presentation: Presented in part at the Annual Meeting of the American Society of Pediatric Otolaryngology, Austin, TX, May 18-21, 2017.

The authors included in this manuscript have no financial interests in the subject matter or materials discussed in this manuscript.
} 
Grossly, the submandibular mass was a well-circumscribed, unencapsulated lymph node, separate from the submandibular gland. Microscopy demonstrated normal nodal architecture replaced by nests of cells in a whorled pattern within a myxomatous background with infrequent mitotic figures (3/10 HPF) and no atypical mitotic figures. These cells stained largely positive for NKI-C3, smooth muscle actin (SMA), CD10, neuron-specific enolase (NSE), and PGP9.5 (Figure 2). Staining was negative for S-100, GFAP, pan-keratin, epithelial membrane antigen (EMA), HMB45, and CD34. The nasal nodule also contained small nests of the same cells. Of three perifacial lymph nodes evaluated, one was replaced by tumor. None of the remaining resected cervical lymph nodes contained tumor $(0 / 13)$. Due to the unusual nature of the pathologic findings the specimen was sent for independent pathology review at Brigham and Women's Hospital which confirmed the diagnosis of cellular neurothekeoma.

The patient underwent three further Mohs type resections of the primary tumor site. Permanent pathologic analysis was used to determine complete resection. Multidisciplinary institutional review did not recommend adjuvant therapy. Two years following the final resection the patient had no evidence of disease.

\section{Discussion}

Neurothekeoma is a rare, reputedly benign tumor of childhood, most commonly presenting as a slow-growing, painless papule or nodule in the first three decades of life with a slight female predominance. ${ }^{2-4}$ First described as a nerve sheath myxoma in 1969 by Harkin $^{3}$, the term neurothekeoma was first published in $1980^{4}$. There are multiple histologic subtypes including myxoid, cellular, and mixed. It is believed that myxoid, or "classic", neurothekeoma is derived from nerve sheath cells. ${ }^{4}$ These are described as nonencapsulated proliferations of spindled and epithelioid cells in a myxoid matrix staining positive for S-100 and GFAP. ${ }^{2}$

Cellular neurothekeoma has variable histology and presumably is derived from dermal or perineural lineage. ${ }^{1}$ These tumors are S-100 negative, stain positive for NKI-C3, NSE, and SMA, and commonly contain mitotic figures. ${ }^{1,5}$ Cellular neurothekeoma can be locally invasive with perineural and vascular invasion and occasional local recurrence, but there is no previously documented locoregional metastasis. ${ }^{1}$ A similar entity, plexiform fibrohystiocytic tumors, have rarely been reported to metastasize. ${ }^{6,7}$ Cellular neurothekeomas have been proposed to be a variant of fibrohystiocytic tumors; ${ }^{8}$ however, Hornick and Fletcher state the two lesions have different anatomic distributions, different morphologic appearance, and "shared positivity for relatively ubiquitous antigens such as SMA and NKI-C3" in the largest series of cellular neurothekeomas reported. ${ }^{1}$ In our case, two independent reviews of the pathology confirmed the diagnosis of cellular neurothekeoma.

Surgical excision with microscopic negative margins is the mainstay of treatment. ${ }^{2,5,9}$ In a case series of 178 neurothekeomas, recurrence occurred in approximately $15 \%$ of patients, all with incomplete initial resection. ${ }^{2}$ There have been reports of multiple neurothekeomas presenting concurrently ${ }^{10-12}$ and without imaging prior to the first surgery we cannot rule 
out concurrent lesions in this case. This remains the first report of neurothekeoma within lymph nodes, and the locoregional location of multiple affected lymph nodes combined with delayed clinical presentation of the neck mass support the hypothesis of metastatic spread from the original lesion. It is our theory that the cervical lesions described in this case were metastases due to residual tumor following resection.

This case and review of the current literature support the recommendation that patients with cellular neurothekeoma of the head and neck undergo complete surgical excision with confirmed negative microscopic margins on permanent histology. In the case of positive margins, we further recommend the approach taken here of multiple small "Mohs type" resections. This allows for shorter anesthesia exposure in pediatric patients and formal pathology review of permanent histology and immunohistochemistry. Finally, this case exhibits the importance of long-term monitoring for regional metastasis in cellular neurothekeoma patients with positive margins.

\section{Acknowledgements:}

Carrie Capri and Eden Palmer for manuscript and figure preparation.

Funding Source: Seattle Children's Hospital Guild Funding Focus Award and NIH RO1 NS092772, NIHMS-ID 905693

\section{References:}

1. Hornick JL, Fletcher CD. Cellular neurothekeoma: detailed characterization in a series of 133 cases. The American journal of surgical pathology. 2007;31(3):329-40. Epub 2007/02/28. doi: 10.1097/01.pas.0000213360.03133.89. [PubMed: 17325474]

2. Fetsch JF, Laskin WB, Hallman JR, Lupton GP, Miettinen M. Neurothekeoma: an analysis of 178 tumors with detailed immunohistochemical data and long-term patient follow-up information. The American journal of surgical pathology. 2007;31(7):1103-14. Epub 2007/06/27. doi: 10.1097/PAS. 0b013e31802d96af. [PubMed: 17592278]

3. Harkin JC, Reed RJ. Tumors of the peripheral nervous system Atlas of Tumor Pathology. Washington DC: Armed Forces Institutes of Pathology; 1969 p. 60-4.

4. Gallager RL, Helwig EB. Neurothekeoma--a benign cutaneous tumor of neural origin. American journal of clinical pathology. 1980;74(6):759-64. Epub 1980/12/01 [PubMed: 7446487]

5. Yun SJ, Park HS, Lee JB, Kim SJ, Lee SC, Won YH. Myxoid Cellular Neurothekeoma: A New Entity of S100-Negative, CD68-Positive Myxoid Neurothekeoma. Annals of dermatology. 2014;26(4):510-3. Epub 2014/08/22. doi: 10.5021/ad.2014.26.4.510. [PubMed: 25143683]

6. Moosavi C, Jha P, Fanburg-Smith JC. An update on plexiform fibrohistiocytic tumor and addition of 66 new cases from the Armed Forces Institute of Pathology, in honor of Franz M. Enzinger, MD. Annals of diagnostic pathology. 2007;11(5):313-9. Epub 2007/09/18. doi: 10.1016/j.anndiagpath. 2007.01.003. [PubMed: 17870015]

7. Remstein ED, Arndt CA, Nascimento AG. Plexiform fibrohistiocytic tumor: clinicopathologic analysis of 22 cases. The American journal of surgical pathology. 1999;23(6):662-70. Epub 1999/06/12. [PubMed: 10366148]

8. Zelger BG, Steiner H, Kutzner H, Maier H, Zelger B. Cellular 'neurothekeoma': an epithelioid variant of dermatofibroma? Histopathology. 1998;32(5):414-22. Epub 1998/06/25. [PubMed: 9639116]

9. Kose D, Ugras S, Harmankaya I, Ciftci I, Koksal Y. Neurothekeoma in childhood: a benign tumor mimicking malignant disease. The Turkish journal of pediatrics. 2014;56(2):208-11. Epub 2014/06/10. [PubMed: 24911861] 
10. Garcia-Gutierrez M, Toussaint-Caire S, Gonzalez-Sanchez P, Ortiz-Hidalgo C. Multiple desmoplastic cellular neurothekeomas localized to the face of a 16-year-old boy. The American Journal of dermatopathology. 2010;32(8):841-5. Epub 2010/12/09. [PubMed: 21137111]

11. Gill P, Abi Daoud MS. Multiple cellular neurothekeomas in a middle-aged woman including the lower extremity: A case report and review of the current literature. Journal of cutaneous pathology. 2019;46(1):67-73. Epub 2018/10/03. doi: 10.1111/cup.13366. [PubMed: 30270462]

12. Mahalingam M, Alter JN, Bhawan J. Multiple cellular neurothekeomas--a case report and review on the role of immunohistochemistry as a histologic adjunct. Journal of cutaneous pathology. 2006;33(1):51-6. Epub 2006/01/31. doi: 10.1111/j.0303-6987.2006.00400.x. [PubMed: 16441413] 


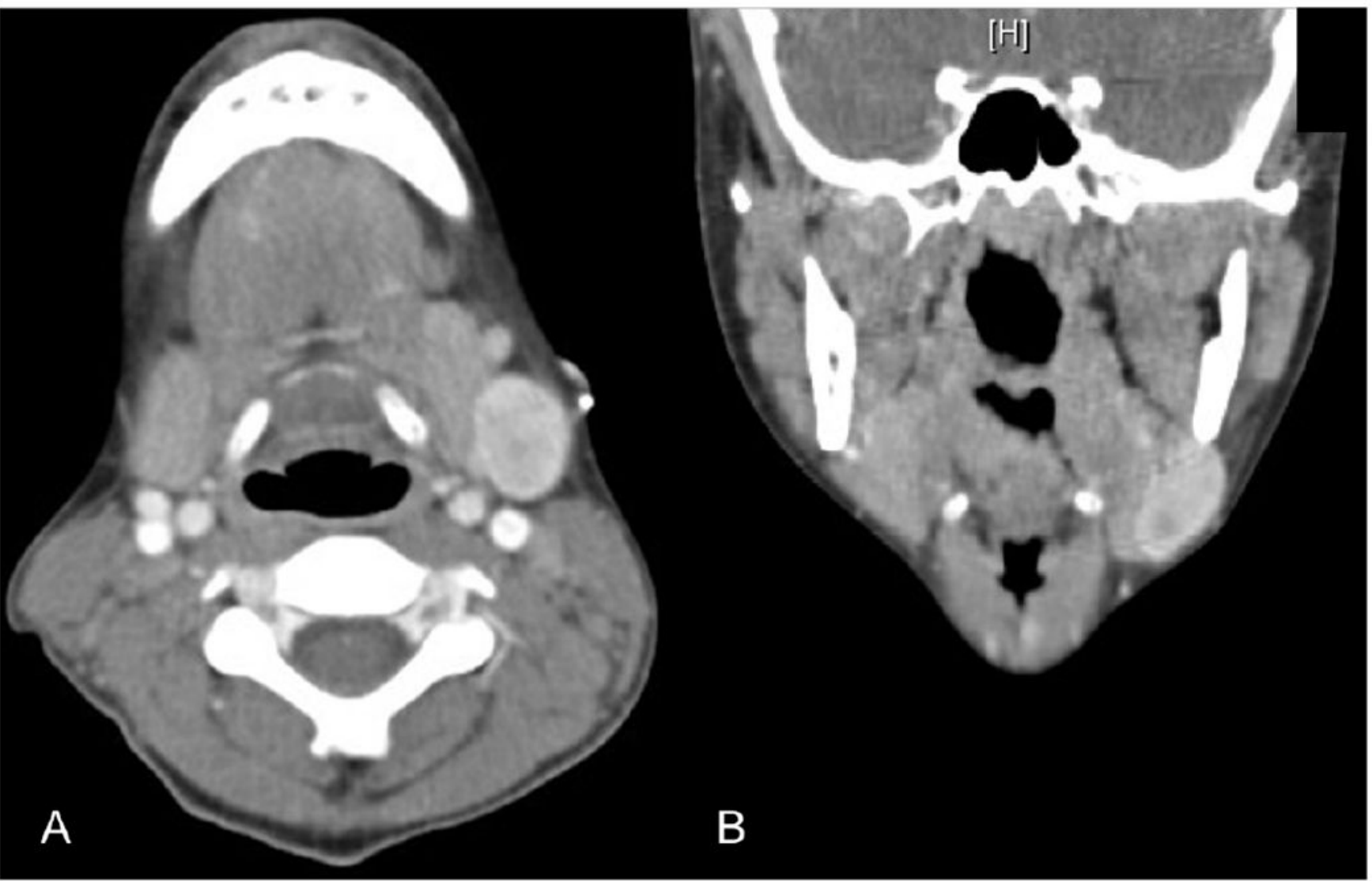

Figure 1:

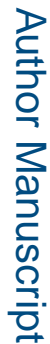

Axial and coronal CT with contrast of left submandibular mass. 

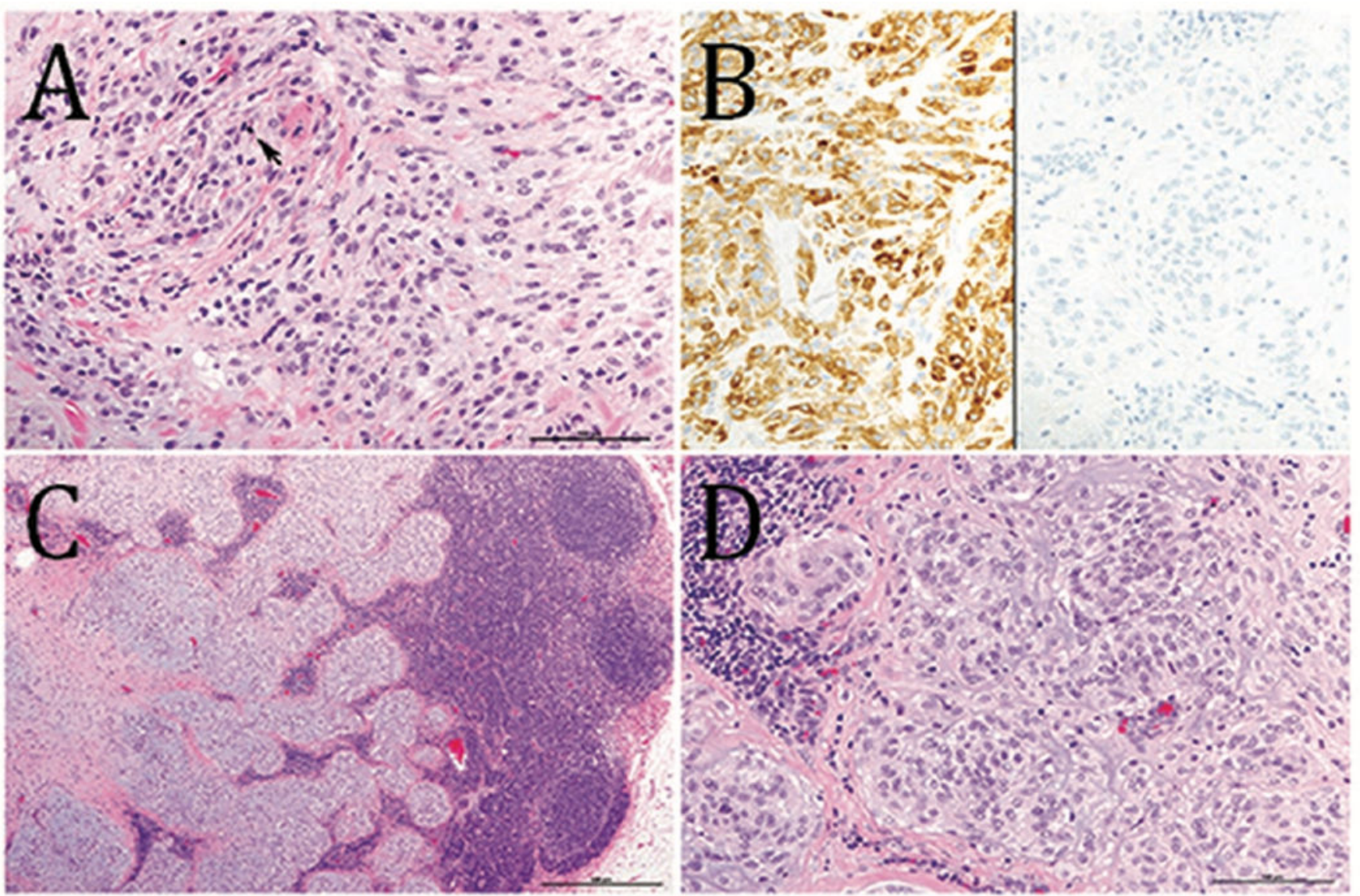

Figure 2:

Histologic evaluation of primary nasal vestibule tumor and excised lymph nodes A) Cellular neurothekeoma in the left nasal cavity, consisting of bland, histiocytoid cells with scattered mitotic figures (arrow). B) The tumor cells stain intensely for CD10 (left panel) and are negative for HMB45 (right panel). C) Metastatic tumor in a peri-facial lymph node. D) Higher power demonstrates nests of tumor cells morphologically similar to the primary lesion. 